Joyce Rodvie M. Sagun, MD

Emmanuel Tadeus S. Cruz, MD

Department of Otorhinolaryngology

Head and Neck Surgery

Quezon City General Hospital and Medical Center
Correspondence: Dr. Emmanuel Tadeus S. Cruz Department of Otorhinolaryngology Head \& Neck Surgery Quezon City General Hospital and Medical Center

Seminary Road, Muñoz, Quezon City 1106 Philippines

Phone: (632) 8630800 local 401

Fax: (632) $9207081 ; 9206270$

Email:emancrz@yahoo.com

(contact details may be published)

The authors declare that this represents original material, that the manuscript has been read and approved by all the authors, that the requirements for authorship have been met by each author, and that each author believes that the manuscript represents honest work.

Disclosures: The authors signed disclosures that there are no financial or other (including personal) relationships, intellectual passion, political or religious beliefs, and institutional affiliations that might lead to a conflict of interest

Presented at the Quezon City General Hospital 33rd Interesting Case contest (3rd place). 17 August 2016 QCGH, Muñoz, Quezon City.

\section{Bilateral Cricoarytenoid Joint Ankylosis with a Perplexing Etiology}

Immobility, fixation or paralysis of the vocal folds is an ominous sign when encountered in the clinics. This may be due to a variety of diseases, lesions, injuries or vascular compromise which may affect the integrity and physiologic mechanism of the vocal folds. The common etiologies include infectious processes such as laryngeal or pulmonary tuberculosis (PTB), malignancy or neoplasms, central problems such as cerebrovascular accidents (CVA), stroke and others. ${ }^{1,2,3}$ The problem should be addressed immediately because this potentially life threatening and imminent narrowing of the glottic opening may lead to respiratory distress. Vocal fold paralysis due to compression of the recurrent laryngeal nerve from PTB and laryngeal cancer are perennially seen in clinical practice, but immobility of the vocal folds due to cricoarytenoid joint fixation or ankylosis is seldom seen and appreciated.

Hence, we present a case of bilateral cricoarytenoid joint ankylosis and discuss its etiology, pathophysiology, differential diagnoses, ancillary procedures and management.

\section{CASE REPORT}

A 60-year-old man was admitted for the first time because of difficulty of breathing and stridor. One week prior to admission, he started to experience difficult breathing associated with productive cough and colds. He consulted in a primary private hospital and was managed as a case of bronchial asthma in exacerbation. Nebulization with salbutamol afforded temporary relief.

A few hours prior to admission, difficulty of breathing and productive cough worsened, prompting emergency room consult. He was referred to us for further evaluation of stridor.

The patient had no diabetes mellitus, hypertension or allergies to food and drugs. He was diagnosed with refractory bronchial asthma during childhood and had frequent hospitalizations for pulmonary infections. He had no maintenance medication for bronchial asthma and was nebulized with salbutamol during exacerbations. He had PTB and completed six months' anti-TB medications in 2013. The patient claimed that he had no dyspneic episodes during routine daily activities or upon exertion. No history of hoarseness or joint pain was noted either. A golf caddy, he was a previous 15-pack-year smoker, occasional alcoholic beverage drinker and denied use of illicit drugs.

Upon admission, the patient was awake, coherent, not in cardiorespiratory distress. Blood pressure was $110 / 70 \mathrm{mmHg}$, pulse rate was 74 /minute, respiration was tachypneic at 24 cycles per minute, afebrile. Ear examination showed normal pinnae, no tragal tenderness, patent external auditory canals with no discharge and $80-90 \%$ dry central perforations of both tympanic membranes. Anterior rhinoscopy, nasal endoscopy and the oral cavity examination 
were unremarkable. Head and neck examination showed no cervical lymphadenopathy or palpable mass.

Video laryngoscopy showed both vocal folds were immobile and fixed in paramedian position upon inspiration with a 1-2 mm glottic opening and no mass or lesion appreciated. (Figure 1)

The initial impression was impending upper airway obstruction secondary to bilateral vocal fold paralysis. Under general anesthesia, direct laryngoscopy revealed no mass or lesion on both vocal folds and passive mobility test demonstrated resistance and limitation of lateral rotation and movement of the arytenoids on both sides. (Figure 2) The vocal folds did not abduct on lateral retraction of the arytenoids. Tracheostomy was performed and he was discharged after a few days.

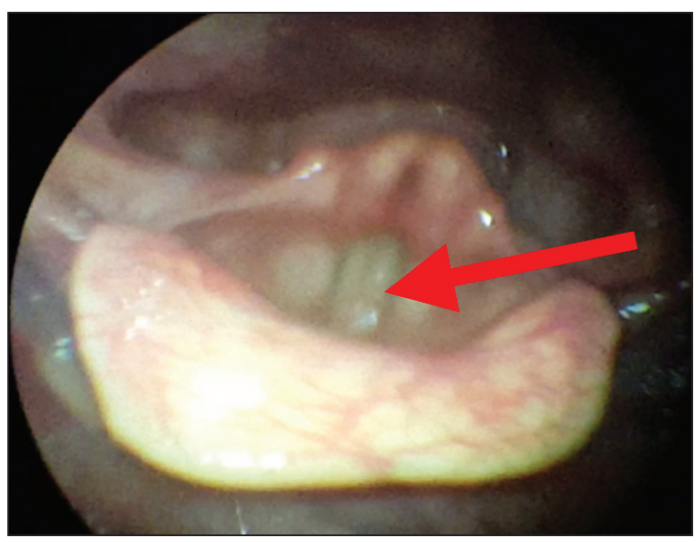

Figure 1. Video laryngoscopy using a $70^{\circ}$ rigid scope showed vocal folds fixed in paramedian position with 1-2 mm glottic opening on inspiration (arrow).
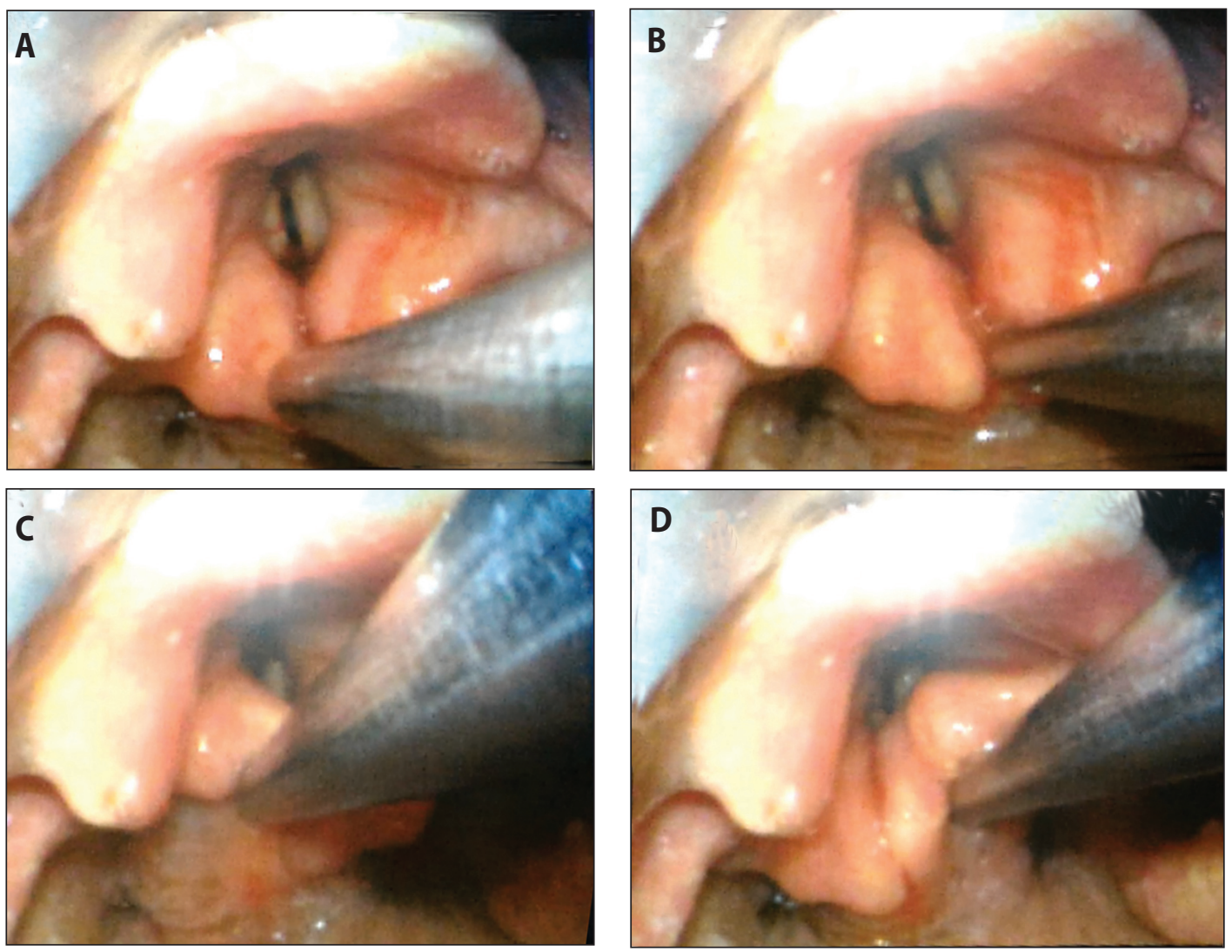

Figure 2. Direct laryngoscopy and passive mobility test using a $3 \mathrm{~mm}$ laryngeal suction tip showing limitation of movement of the cricoarytenoid joints. Lateralization of the (A) left arytenoid and (B) right arytenoid, with noted fixation at midline; Inspection of the left (C) and right (D) cricoarytenoid area with no noted adhesions or swelling. 


\section{FEATURED GRAND ROUNDS}

A subsequent laryngeal electromyography (EMG) study showed no signs of myopathy or acute or chronic denervation changes of the thyroarytenoid muscles and rheumatoid factor was normal. At this point, bilateral cricoarytenoid fixation or ankylosis was considered and posterior interarytenoid web and bilateral vocal fold paralysis were ruled out.

We recommended a lateralization procedure such as unilateral arytenoidectomy with cordectomy. The patient is currently well while he and his family are still contemplating whether he will undergo the surgical procedure.

\section{DISCUSSION}

Respiratory stridor is always considered an ominous sign which implies upper airway obstruction. If severe, stridor may compromise breathing and in some instances is life threatening and a telltale sign of imminent danger requiring immediate endotracheal intubation. Stridor is a musical, high-pitched sound which may be elicited in the presence of laryngeal and upper tracheal obstruction while wheezes are defined as high-pitched, continuous, adventitious lung sounds. ${ }^{4,5}$

Stridor may be due to several reasons such as immaturity of the laryngeal structures seen in laryngomalacia in newborns, laryngeal infection, foreign body in the airway and chronic obstructive pulmonary disease. ${ }^{3,6}$ This may be the reason why bronchial asthma was entertained in the clinical course of our patient and initially at the emergency room. It is unfortunate that despite the non-responsiveness of bronchial asthma to medical therapy and persistence of stridor, no ENT referral to evaluate the upper airway was made until recently. It should be emphasized that patients who develop stridor need to be evaluated by otolaryngologists specifically to ascertain the status of the vocal folds, which in this case turned out to be fixed or ankylosed, a condition which is rarely seen and encountered in clinical practice.

Among the differential diagnoses considered in this case were laryngeal cancer, vocal fold paralysis, interarytenoid web and arthritis. $7,8,9$

Initially, laryngeal cancer was entertained because of his age, however no mass or suspicious lesion was appreciated on video laryngoscopy and this was ruled out. Because the vocal folds were immobile and fixed in paramedian position upon inspiration, bilateral vocal fold paralysis was considered with the etiology to be determined.

Vocal fold paralysis occurs when nerve impulses to the laryngeal muscles are disrupted in case of CVA or stroke, recurrent nerve injury after thyroid surgery or compression of the inferior laryngeal nerve due to pulmonary TB or lung cancer. ${ }^{8,11}$ On the other hand, vocal fold fixation occurs when movement of the cricoarytenoid joint is compromised in cases of rheumatoid arthritis provided that the innervation is intact. ${ }^{10,11}$

Another common differential diagnosis which may be entertained is laryngeal TB in which nodular lesions may be seen in the vocal folds, granulation tissues are usually present in the posterior commissure and histopathology shows Langhans cells and caseation necrosis. ${ }^{8}$ Paralysis is oftentimes unilateral due to compression of the recurrent laryngeal nerve from apical PTB. Although the patient has a history of TB, he was asymptomatic and close examination of the vocal folds revealed no lesions except for bilateral fixation, and this was ruled out.

Direct laryngoscopy (DL), the gold standard in the evaluation of laryngeal anatomy especially when dealing with the vocal folds, ${ }^{3}$ showed smooth, normal-looking vocal folds with no lesions. The passive mobility test is done to differentiate vocal fold paralysis from cricoarytenoid ankylosis by retracting or pushing the arytenoid laterally. If there is limitation of rotation and movement of the arytenoid laterally and the vocal folds do not abduct, then cricoarytenoid ankylosis or fixation is considered. On the contrary, if the arytenoid rotates and abducts laterally when retracted by forceps, then vocal fold paralysis is considered. ${ }^{1,6}$ Hence, because there was limitation of rotation and movement of the arytenoids, cricoarytenoid joint fixation was entertained and vocal fold paralysis was ruled out.

Interarytenoid web was excluded because the vocal folds had no mucosal adhesions, synechiae or any scarring within the posterior portion of the glottis. In addition, although the patient's glottic opening was restricted, no difficulty was encountered during endotracheal intubation since a smaller caliber tube was used.

To further confirm the diagnosis of cricoarytenoid fixation, laryngeal electromyography (EMG) revealed no paralysis of the thyroarytenoid muscles with no signs of myopathy and acute or chronic denervation, making bilateral vocal fold paralysis unlikely in this case. Laryngeal EMG is indicated to determine the integrity of the laryngeal muscles and innervation especially in cases of vocal fold paralysis. ${ }^{11}$ In postthyroidectomy patients, laryngeal EMG is done 6 months after surgery to determine if the laryngeal nerve injury may recover or is irreversible. The 6-month waiting period is to allow swelling or inflammation to subside and to observe whether the injured nerve will recover prior to further intervention. ${ }^{12}$

The findings on direct laryngoscopy, passive mobility test and laryngeal electromyography clearly favor the diagnosis of cricoarytenoid joint ankylosis. Other ancillary procedures such as a CT scan may show sclerosis of the arytenoids ${ }^{1,11}$ in elderly patients and videostroboscopy may be useful in determining the relative vertical height and tension of the vocal folds for assessing the cricoarytenoid 


\section{FEATURED GRAND ROUNDS}

function. ${ }^{1}$ A CT scan was not done because there was no palpable neck mass and no other lesion was entertained that would warrant CT imaging. Videostroboscopy may help and may further show and magnify the movement of the vocal folds for observation however, the findings seen on direct laryngoscopy and laryngeal EMG were deemed enough to support and confirm the diagnosis.

The patient may be classified under type IV posterior glottic stenosis - congenital or acquired bilateral cricoarytenoid fixation with or without interarytenoid scarring - based on the classification by Bogdasarian and Olson which was later modified by Irving and associates. ${ }^{3}$ Interarytenoid web and scarring presents as bilateral impaired abduction but adduction is normal and patients affected tend to have a normal voice while the main presenting symptom is airway compromise. In cricoarytenoid joint ankylosis, adduction and abduction of the vocal folds are limited. ${ }^{3}$ As previously mentioned, to distinguish cricoarytenoid joint ankylosis from vocal fold paralysis, palpation of the cricoarytenoid joint on rigid endoscopy and laryngeal EMG are necessary for definitive diagnosis. ${ }^{6}$

The patient's voice was normal because the vocal folds approximate each other with a 1 to $2 \mathrm{~mm}$ glottic opening while no history of aspiration was apparent because the vocal folds are fixed in paramedian position which may prevent fluid from entering the larynx during swallowing. Although the patient's voice is normal, respiration is compromised manifested as stridor and difficulty of breathing requiring tracheostomy.

In contrast, patients with acute or recent unilateral vocal fold paralysis in post-thyroidectomy or post-CVA (stroke) conditions may initially manifest with aspiration. This is because the vocal fold assumes an intermediate position in which the glottic opening is relatively wider compared with the paramedian position. In a few months' time, the paralyzed fold will compensate, move medially, and assume a paramedian position and aspiration may eventually resolve. ${ }^{13}$

Cricoarytenoid ankylosis has several etiologies which include arthritides, bacterial infection and trauma. Rheumatoid arthritis may account for numerous clinical diagnoses of cricoarytenoid ankylosis. ${ }^{2}$ Other causes include gout, Reiter Syndrome and ankylosing spondylitis. Some anecdotal evidence suggests a mump-associated laryngeal arthritis and fixation secondary to radiation therapy. ${ }^{2,8}$ Bacterial involvement of the joint space with infectious microorganisms such as streptococcal species with resultant ankylosis is also well established. ${ }^{8}$ External and direct laryngeal trauma may also result in cricoarytenoid joint injury. ${ }^{8}$ Documented and retrospective studies suggest intubation-related joint injury and posterior or anterior arytenoid displacement secondary to the distal tip of the endotracheal tube engaging the arytenoid during intubation. ${ }^{8}$ Traumatic obstetric delivery using forceps and postpartum newborn care through vigorous cleansing and suctioning the mouth and pharynx of the newborn are also mentioned in the literature. ${ }^{11}$ Posterior dislocation resulting from extubation with a partially inflated endotracheal tube cuff is another probable cause., 8 Another potential etiology is arytenoid chondritis secondary to prolonged endotracheal intubation which results in fibrosis. ${ }^{8,16}$ Reviewing the patient's history, however, showed no history of trauma, previous intubation, signs and symptoms of arthritis and serious laryngeal infections. The patient was delivered via normal spontaneous delivery by a traditional birth attendant ("hilot") and no apparent respiratory distress or postpartum hospitalization was known of by the patient.

Cricoarytenoid ankylosis is usually associated with cases of rheumatoid arthritis with 17 to $33 \%$ incidence among RA patients. ${ }^{9}$ House et al. in 2010 described approximately $0.1 \%$ incidence of cricoarytenoid joint ankylosis in endotracheal intubations. ${ }^{16}$ Most cases of vocal fold immobility seen under the service is secondary to vocal fold paralysis due to cerebrovascular accident (stroke), pulmonary problems such as PTB or laryngeal malignancy and to our knowledge, this is the first reported case of cricoarytenoid joint ankylosis in our institution.

Chronic cricoarytenoid joint ankylosis may be mistaken for asthma or chronic bronchitis, with symptoms of dyspnea, hoarseness or stridor. ${ }^{3}$ In rheumatoid arthritis, laryngoscopy may show rough and thick mucosa and narrowed glottic chink which were contrary to the recent endoscopic findings. If the etiology is bacterial, there is direct involvement of the joint space with infectious agents such as streptococcal species which leads to scarring and thickening of the cricoarytenoid joints. ${ }^{8}$ Airway compromise occurs most commonly in patients with long-standing cricoarytenoid ankylosis and laryngeal stridor has been described as the sole presentation of the disease as manifested in this case. ${ }^{8,14,17}$ To rule out RA in this case, rheumatoid factor (RF) was done with negative results.

Finally, when it comes to upper airway obstruction, the glottic opening or opening of the vocal folds should be thoroughly evaluated. The normal glottic opening in newborns opens approximately $4 \mathrm{~mm}$ in a lateral direction. Congenital subglottic stenosis is defined as a subglottic diameter of less than $4 \mathrm{~mm} .{ }^{13}$ In retrospect, it may be presumed that the patient's glottis may not be seriously compromised since birth because he was able to thrive and breathe with no apparent difficulty. It may be conjectured that narrowing of the glottic opening occurred only later in life. Although asymptomatic, rheumatoid factor was negative, and the etiology of the patient's ankylosis remains perplexing and elusive.

The management of cricoarytenoid ankylosis includes tracheostomy to address the upper airway obstruction. Surgical management 
FEATURED GRAND ROUNDS

includes open arytenoidectomy, arytenoidpexy and endoscopic arytenoidectomy or transverse cordectomyandall have theiradvantages and disadvantages. ${ }^{6,11,16}$ These are lateralization procedures which aim to widen the glottic opening and wean the patient from tracheostomy afterwards.

In closing, when bronchial asthma remains refractory to treatment, the physician should not hesitate to refer to otolaryngologists to rule out other probable upper airway pathologies. Although rare, ankyloses of the cricoarytenoid joint should be considered especially when the movement of the vocal folds is compromised. Although direct laryngoscopy, passive mobility tests and laryngeal EMG are indispensable in clinching the diagnosis, the clinical history is important in determining etiology which in this case remains elusive and perplexing.

\section{ACKNOWLEDGEMENTS}

We acknowledge Antonio G. Talapian, MD, FPSOHNS, Francisco A. Victoria, MD, FPSOHNS, and Emmanuel Samson, MD, FPSOHNS, for their scientific advise.

\section{REFERENCES}

1. Woo Peak M. Laryngeal Trauma in Woo Peak Stroboscopy. $1^{\text {st }}$ ed. California: Plural Publishing Inc. 2010. p. 221-226.

2. Bryson PC, Buckmire RA. Medscape. Arytenoid Fixation. [Updated 2016 Mar 30; cited 2013 Apr]. Retrieved from: https://emedicine.medscape.com/article/866384-overview\#a6.

3. Albert D, Boardman S, Soma M. Evaluation and Management of the Stridulous Child. In Flint PW, Haughey BH, Lung VJ, Niparko JK, Richardson MA, Robbins T, et al (editors). Cummings Otorhinolarynglogy Head and Neck Surgery. $5^{\text {th }}$ ed. Philadel phia: Mosby Elsevier. 2010. p. 28962897.

4. Lechtzin NM. Stridor. Merck Manual Professional. 2014 [cited 2014 Apr]. Retrieved from Merck Manuals: http://www.merckmanuals.com/professional/pulmonary-disorders/symptoms-ofpulmonary-disorders/stridor.

5. Lechtzin NM. Wheezing. Merck Manual Professional. 2014 [cited 2014 Apr]. Retrieved from Merck Manuals: http://www.merckmanuals.com/professional/pulmonary-disorders/symptoms-ofpulmonary-disorders/wheezing.

6. Jackson C, Jackson CL. Direct Laryngoscopy in Bronchoesophagology. $5^{\text {th }}$ ed. Philadelphia: W.B. Saunders. 1950. p. 116-117

7. Cooper R. Extubation and Changing Endotracheal Tubes. In Benumof J, Hagberg CA (editors). Benumof's Airway Management: Principles and Practice. $2^{\text {nd }}$ ed. Philadelphia: Mosby Elsevier. 2007. p. 1160.

8. Heman-Ackah Y, Kelleher K, Sataloff R. Inferior Glottic Ridges That Prevent Vocal Cord Closure. In Sataloff RT, Chowdhury F, Joglekar S, Hawkshaw MJ (editors). Atlas of Endoscopic Laryngeal Surgery. $1^{\text {st }}$ ed. New Delhi: Jaypee Brothers Medical Publishers. 2011. p. 45

9. Kamanli A, Gok U, Sahin S, Kaygusuz I, Ardicoglu O Yalcin S. Bilateral cricoarytenoid joint involvement in rheumatoid arthritis: a case report. Rheumatology (Oxford). 2001 May; 40 (5): 593-594. PMID: 11371675.

10. Zakaria HM, Al Awad NA, Al Kreedes AS, Al-Mulhim AM, Al-Sharway MA, Hadi MA, et al. Recurrent laryngeal nerve injury in thyroid surgery. Oman Med J. 2011 Jan; 26(1): 34-38. DOI: 10.5001/omj.2011.09; PMID: 22043377 PMCID: PMC3191623.

11. Remacle M, Sandhu G. Bilateral Vocal Fold Immobility. In Oswal V, Remacle M, Jovanvic S, Zeitels SM, Krespi JP, Hopper C (editors). Principles and Practice of Lasers in Otorhinolaryngology and Head and Neck Surgery. $2^{\text {nd }}$ ed. Amsterdam: Kugler Publication. 2014. p. 250-251.

12. Chauhan A, Badhwar S, Patel M, Tiwari S. Post - Intubation Bilateral Arytenoid Dislocation with Acute Respiratory Distress. J Anaesth Clin Pharmacol. 2009 Aug; 25(3):361-62.

13. Banovetz J. Benign Laryngeal Disorders. In Adams GL, Boies LR (editors). Boies Fundamentals of Otolaryngology - A Textbook of Ear, Nose, and Throat Diseases. $6^{\text {th }}$ ed. Singapore:W.B. Saunders. 1989. p. 406-408.

14. Stojanovic SP, Zivic L, Stojanovic J, Belic B. Total fixation of cricoarytenoid joint of a patient with rheumatoid arthritis and Hashimoto thyroiditis. Srp Arh Celok Lek. 2010 Mar-Apr; 138(3-4): 230-2. PMID: 20499506.

15. Hamdan AL, Sarieddine D. Laryngeal Manifestations of Rheumatoid Arthritis. Hindawi Autoimmune Diseases. 2013; 2013: 4-6. DOI:10.1155/2013/103081.

16. Polisar IA, Burbank B, Levitt LM, Katz HM, Morrione TG. Bilateral midline fixation of cricoarytenoid joints as a serious medical emergency. JAMA. 1960; 172(9): 901-906. DOI:10.1001/ jama.1960.03020090013003.

17. Burkey B, Goudy S, Rohde S. Airway Control and Laryngotracheal Stenosis in Adults. In Snow JB, Ballenger JJ (editors). Ballenger's Manual of Otorhinolaryngology Head and Neck Surgery. 17th ed. Ontario: BC Decker. 2009. p. 911. 